\title{
Детектор нейтронов на основе кристаллов TIInSe, интеркалированных изотопом лития
}

\author{
() И.В. Алексеев, Е.А. Горемычкин, Н.А. Гундорин, \\ А.В. Петренко, И.Л. Сашин
}

Объединенный институт ядерных исследований, 141980 Дубна, Россия

E-mail: aliv2@yandex.ru

(Получена 11 января 2016 г. Принята к печати 21 ноября 2016 г.)

Исследовалась возможность увеличения чувствительности детектора нейтронов на основе полупроводника $\mathrm{TlInSe}_{2}$ путем введения в кристалл изотопа лития ${ }^{6} \mathrm{Li}$. Введение проводилось методом электрохимической интеркаляции из водных и неводных растворов $\mathrm{LiCl}$, а также из расплава эвтектики $\mathrm{LiCl}-\mathrm{KCl}$. Показана эффективность интеркалирования электрохимическим методом из раствора $\mathrm{LiCl}$ в пропиленкарбонате, проводимого вдоль оси „с“ кристалла (вдоль цепочек сильной связи). Достигнутая концентрация введенного лития составила $(1-1.2) \cdot 10^{21} \mathrm{~cm}^{-3}$, что примерно вчетверо увеличило чувствительность детектора. Показано хорошее согласие между рассчитанным и фактическим значением чувствительности детектора.

DOI: $10.21883 /$ FTP.2017.06.44555.8170

\section{1. Введение}

В работе [1] описан детектор тепловых нейтронов на основе чувствительного к $\gamma$-излучению кристалла полупроводникового соединения TIInSe 2 . Действие детектора основывалось на ионизации кристалла захватным $\gamma$-излучением из предусмотренного в корпусе детектора Cd-радиатора.

Цель настоящей работы - увеличение чувствительности детектора [1] посредством введения в $\mathrm{TlInSe}_{2}$ изотопа ${ }^{6} \mathrm{Li}$ и активизации на нем реакции $(n, \alpha)$. Ионизирующая способность электрически заряженных частиц выше, чем у $\gamma$-излучения, с чем и связано ожидаемое увеличение чувствительности детектора. Введение же в кристалл большого количества изотопа ${ }^{6} \mathrm{Li}(\sim 20$ ат\%) может быть осуществлено в анизотропном кристалле $\mathrm{TIInSe} \mathrm{I}_{2}$ методом интеркаляции.

В работе [2] представлены эксперименты по электрохимической интеркаляции (ЭИК) лития в $\mathrm{TlInSe}_{2}$ из водного раствора хлористого лития. Было показано, что ионы лития практически не фиксировались в решетке, а, дрейфуя через образец от контактной грани (грани, находящейся в контакте с электролитом) под действием электрического поля, выделялись на противоположной грани в виде металлического лития. Причиной этого могло быть экранирующее действие избыточного относительно стехиометрии селена, сосредоточенного, как это свойственно анизотропным кристаллам [3], в межцепочечном пространстве кристаллической решетки.

С целью повышения содержания лития в матрице мы апробировали две модификации интеркалирования способ диффузии из расплава [4] и ЭИК из апротонного раствора соли $\mathrm{LiCl}$. В первом случае ионы лития двигаются в кристалле за счет диффузии, а не дрейфа в электрическом поле, и упомянутое выведение интер- калянта из объема образцов TIInSe 2 будет исключено. Во втором случае количество введенного лития может быть повышено благодаря тому, что значительная часть наблюдаемого тока через образец будет обусловлена током катионов $\mathrm{Li}^{+}$, а не ионов $\mathrm{H}^{+}$, конкурирующего с „полезным“ током.

\section{2. Объекты, методы и результаты эксперимента}

Получение кристаллов $\mathrm{TlInSe}_{2}$ описано в [5]. Как известно, соединение $\mathrm{TlInSe}_{2}$ кристаллизуется в анизотропной решетке типа TlSe цепочечной структуры. Для интеркаляции использовался специально не легированный материал $p$-типа проводимости. Образцы изготавливались путем раскалывания монокристаллического слитка по двум взаимно перпендикулярным плоскостям спайности $\{110\}$. После этой операции образцы разрезались перпендикулярно цепочкам в плоскости (001) образивной лентой с образованием кристаллических стержней прямоугольного сечения.

Интеркалирование проводилось на кристаллах $\mathrm{TIInSe}$ с относительно низким удельным сопротивлением $\sim 6 \cdot 10^{5} \mathrm{OM} \cdot \mathrm{cm}$, которые характеризовались повышенной токовой чувствительностью. В таких образцах содержится избыточный относительно стехиометрии селен, примесь которого играет в $p$-TIInSe $\mathrm{T}_{2}$ роль мелкого акцептора [6]. Значительная часть избыточного селена, как уже отмечали, оказывается вытесненной в межцепочечное ван-дер-ваальсовое пространство.

Для интеркалирования методом диффузии была собрана установка, состоящая из кварцевого реактора, через который пропускался аргон и внутри которого располагалась кварцевая же „лодочка“ с расплавом эвтектики двух солей $\left(58.5\right.$ моль $\%{ }^{6} \mathrm{LiCl}+41.5$ моль $\left.\% \mathrm{KCl}\right)$ [7]. 
В расплаве удерживался погруженный в него интеркалируемый образец кристалла $\mathrm{Tl} \mathrm{InSe}_{2}$. Сам реактор находился внутри печи, с помощью которой поддерживалась температура расплава $450^{\circ} \mathrm{C}$ (температура плавления эвтектики $352^{\circ} \mathrm{C}$ ).

Образец выдерживали в расплаве в течение 5-8ч, после чего специальным устройством содержимое „лодочки“ опрокидывали на решетку, в результате чего расплав протекал в поддон, а образец оставался на решетке (последнюю процедуру проводили, не извлекая „лодочку“ из горячей зоны). Далее, после медленного снижения температуры реактора до комнатной, остатки отвердевшей эвтектики $\left({ }^{6} \mathrm{LiCl}+\mathrm{KCl}\right)$ на образце смывали дистиллированной водой.

Несмотря на относительную простоту данного метода, мы вынуждены были отказаться от него, так как при застывании остатков эвтектики на поверхности образца последний расслаивался по плоскостям спайности $\{110\}$ и разваливался на части. В связи с этим мы обратились ко второму средству.

Процесс ЭИК из апротонного электролита с ${ }^{6} \mathrm{LiCl}$ проводился на установке, аналогичной описанной в [2]. В качестве апротонного растворителя был использован пропиленкарбонат (ПКБ) высокой чистоты с удельным сопротивлением $\sim 0.19 \cdot 10^{6} \mathrm{OM} \cdot \mathrm{cM}$.

ЭИК проводили из 0.1 -мольного раствора ${ }^{6} \mathrm{LiCl}$ при комнатной температуре, в режиме постоянного тока с плотностью $0.05-0.06 \mathrm{MA} / \mathrm{cm}^{2}$. Электрическое поле было перпендикулярно цепочкам сильной связи кристалла и имело направление [110]. Апробировалась также и отличная от [2] геометрия интеркалирования, когда электрическое поле было направлено в кристалле вдоль цепочек сильной связи - в направлении [001]. В этом случае плотность тока составляла примерно $0.08 \mathrm{MA} / \mathrm{cm}^{2}$. ЭИК проводилось в 20-25 циклов по 8-10 часов с количеством пропущенного через образец заряда $Q=0.4-0.5$ Кл за цикл.

Явления, которые сопровождали циклы ЭИК кристаллов $\mathrm{TlInSe} \mathrm{S}_{2}$ из водного раствора, - выделение селена на гранях образца и значительное обратимое увеличение проводимости в течение цикла, - наблюдались и теперь. С целью устранения возможных загрязнений поверхности образца и уменьшения влияния поверхности на величину тока интеркаляции, образец после каждых двух-трех циклов промывался в ультразвуковой ванночке.

В случае ЭИК из электролита на основе ПКБ мы наблюдали скопление у контактной грани образца и выпадение в раствор темно-коричневого осадка, содержащего селен. Можно предположить, что мы имеем дело с явлением, подобным электрохимическому растворению металлического анода. Явление было особенно заметным при ЭИК образцов с избыточным относительно стехиометрии содержанием селена. Это явление приводило к эрозийному повреждению контактной грани образцов, что ограничивало длительность процесса ЭИК и количество вводимого за цикл лития.
Следует отметить также уже упоминавшуюся в [2] зависимость характера протекания ЭИК от механического совершенства кристаллических образцов. Если при разделке монокристаллического слитка $\mathrm{TlInSe} \mathrm{S}_{2}$ происходило микроповреждение кристалла, заключающееся в ослаблении связи по какой-то из плоскостей $\{110\}$, то после двух-трех циклов мы наблюдали резкое увеличение проводимости образца в процессе цикла. Потом оказывалось, что в пространство между соответствующими плоскостями спайности с ослабленной связью направлялся основной поток интеркалянта, перемыкавший образец от контактной грани до металлического электрода. После чего становилось возможным и капиллярное проникновение самого электролита в образовавшийся зазор. (Подобное явление имеет место [8] и при ЭИК в кристаллы слоистой структуры). При продолжении ЭИК такого образца он расслаивался, и на открывшихся плоскостях можно было наблюдать белесые следы гидроксида лития.

Таким образом, основным препятствием накопления изотопа лития значительных концентраций в кристаллах TlInSe 2 является наличие механических дефектов в интеркалируемых образцах. Кроме того, из-за вынужденного „мягкого“ режима ЭИК (малые токи интеркаляции) продолжительность работы с каждым образцом затягивалась на многие дни, что также ограничивало возможность достижения значительных концентраций изотопа.

Оптимальной в этом смысле схемой ЭИК оказалась схема, при которой интеркалянт вводится в образец вдоль цепочек сильной связи сразу с двух сторон - от концов к середине. Для этого на образец с примерным размером $10 \times 1.5 \times 1.5$ мм в его центральной части наносился напаиванием индия узкий опоясывающий контакт, к которому крепился электрод. Образец располагался горизонтально, а справа и слева к его торцам $1.5 \times 1.5$ мм подводился специальным устройством электролит. Таким образом, катионы лития дрейфовали от концов к середине образца. Схема позволяла уменьшить в 2 раза плотность тока без уменьшения пропускаемого через ячейку заряда. В последней серии ЭИК расчетная концентрация лития в образцах составляла $(1.0-1.2) \cdot 10^{21} \mathrm{~cm}^{-3}$. Отметим, что количество атомовкомпонентов в кристаллах данного полупроводника составляет $3.6 \cdot 10^{22} \mathrm{~cm}^{-3}$.

\section{3. Обсуждение результатов}

На две противоположные грани интеркалированных литием образцов с примерными размерами $10 \times 1.5 \times 1.5$ мм наносились индиевые контакты. Образованный таким образом чувствительный элемент детектора крепился в алюминиевом корпусе (рис. 1), а два токовых электрода выводились к измерительному блоку. Чувствительный полупроводниковый элемент детектора располагался в цилиндрической части корпуса с длиной 


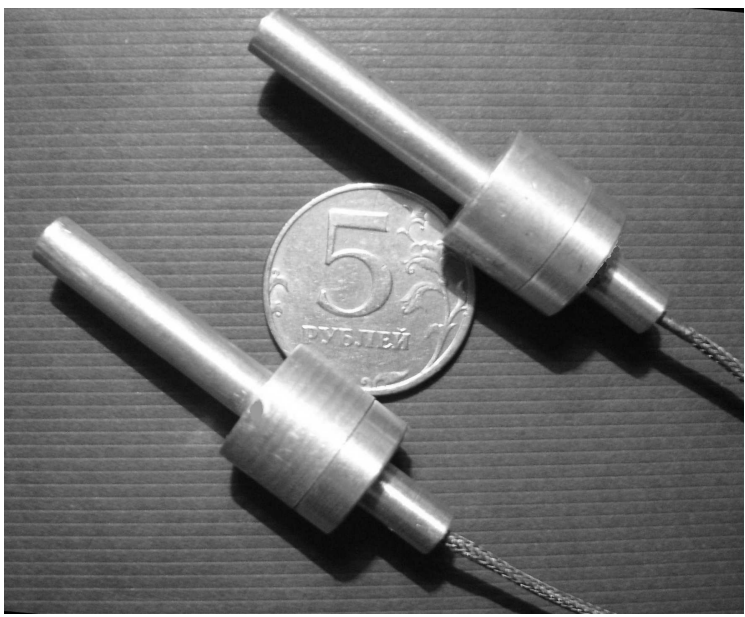

Рис. 1. Внешний вид детекторов.

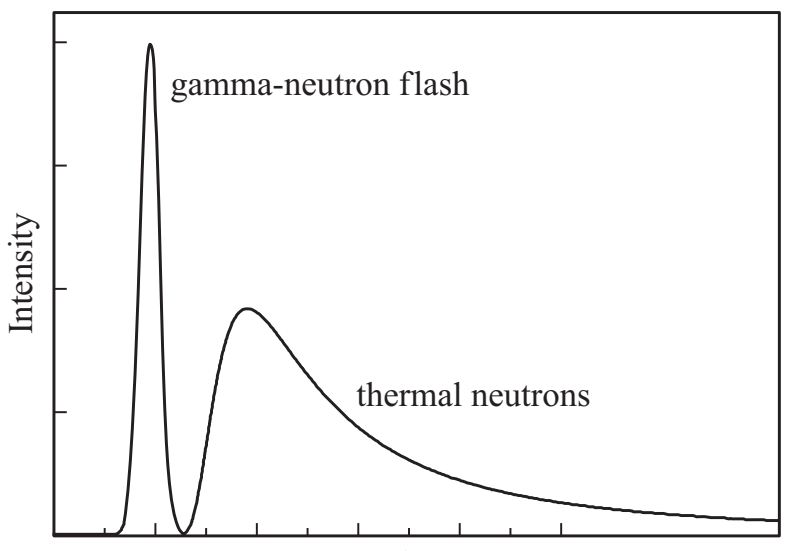

Time

Рис. 2. Схематическое изображение $\gamma$-нейтронного реакторного импульса.

30 мм и диаметром 5 мм. Напряжение питания детектора составляло $9.5 \mathrm{~B}$.

Данный детектор исследовался и тестировался на импульсном реакторе ИБР-2 (ОИЯИ, г. Дубна). Тестирование давало также возможность оценить фактическое содержание лития в интеркалированных кристаллах, которое в силу упоминавшихся причин могло существенно различаться с расчетным.

Схема импульса излучения реактора ИБР-2 изображена на рис. 2. Здесь мы видим короткий импульс $\gamma$-нейтронного излучения и испускаемый одновременно с ним, но растянутый по времени пробега от источника до детектора импульс тепловых нейтронов с непрерывным спектром энергий.

На рис. 3-5 приведены типичные осциллограммы отклика детектора на одном из экспериментальных каналов с 10-метровой времяпролетной базой реактора ИБР-2. Осциллограмма рис. 3 соответствует отклику детектора, в чувствительном элементе которого присутствует ${ }^{6} \mathrm{Li}$, на рис. 4 - отклик детектора в прежнем

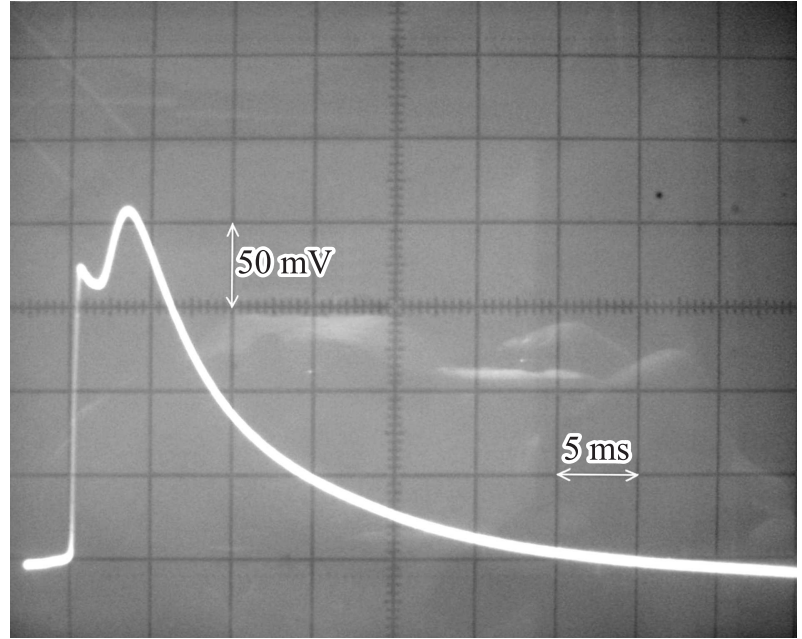

Рис. 3. Осциллограмма сигнала детектора, содержащего литий.

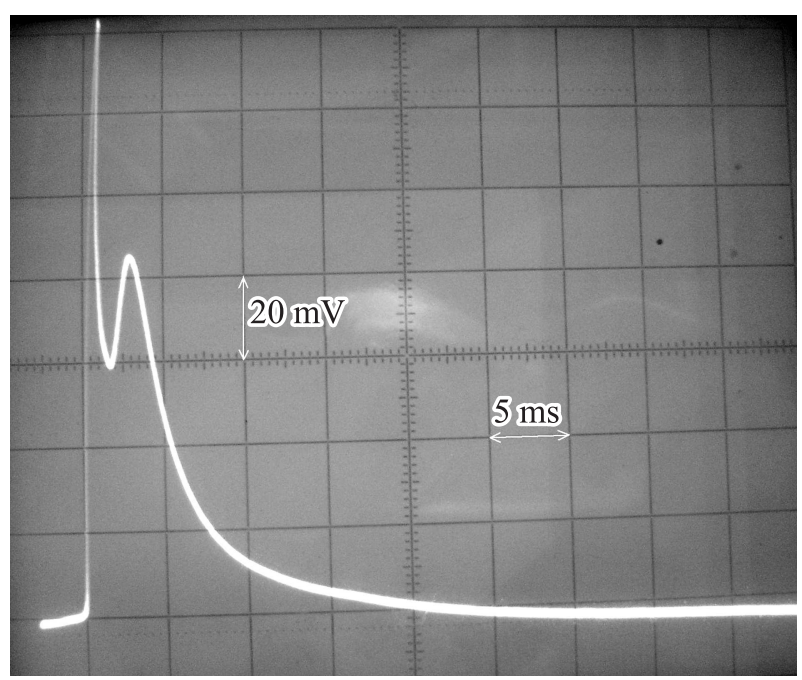

Рис. 4. Осциллограмма сигнала детектора, не содержащего литий.

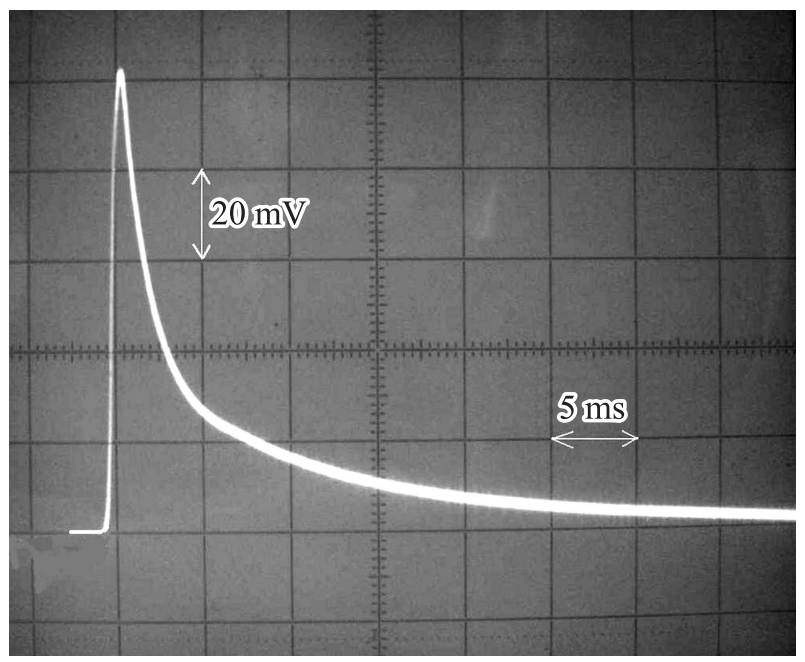

Рис. 5. Осциллограмма детектора (с литием), закрытого экраном из изотопа ${ }^{6} \mathrm{Li}$ (порошок ${ }^{6} \mathrm{LiF}$ с толщиной слоя $2.5 \mathrm{MM}$ ). 
исполнении, без лития. На рис. 5 показана осциллограмма отклика детектора с ${ }^{6} \mathrm{Li}$, закрытого от излучения литиевым экраном, представляющим слой порошка ${ }^{6} \mathrm{LiF}$ толщиной 2.5 мм. Такой экран практически до нуля уменьшает нейтронную компоненту сигнала детектора.

На $\gamma$-компоненте отклика присутствие лития, очевидно, никак не сказывается, а соотношение $\gamma$ - и нейтронной компонент на осциллограммах существенно различается - присутствие лития по меньшей мере вчетверо увеличило нейтронную компоненту в сигнале детектора. Оценка чувствительности детектора к нейтронам дала значение $\sim 1.2 \cdot 10^{-14} \mathrm{~A} \cdot \mathrm{cm}^{-2}$ с/нейтрон.

Абсолютное значение амплитуды нейтронной компоненты, полученное при тестировании детектора, позволяет оценить фактическое содержание изотопа лития в полупроводнике. Если принять, что поток падающих на кристалл нейтронов хорошо сколлимирован, то внутри образца поток ослабляется, поглощаясь на атомах ${ }^{115} \mathrm{In}$ и ${ }^{6} \mathrm{Li}$ по известному закону Бугера:

$$
F(x)=F_{0} \exp \left[-\left(\sigma_{1} n_{1}+\sigma_{2} n_{2}\right) x\right]
$$

а количество нейтронов, поглощенных в образце, равно

$$
\Delta F=F_{0}\left\{1-\exp \left[-\left(\sigma_{1} n_{1}+\sigma_{2} n_{2}\right) d\right]\right\},
$$

где $F_{0}$ и $F(x)$ есть величина падающего потока тепловых нейтронов и этого потока на глубине $x$ в образце, $d-$ толщина образца в направлении нейтронного потока, а $\sigma_{1} n_{1}$ и $\sigma_{2} n_{2}$ - произведения сечения захвата на концентрацию для соответственно изотопа ${ }^{6} \mathrm{Li} \mathrm{и}{ }^{115} \mathrm{In}$.

Индиевая компонента соединения, ослабляющая наряду с изотопом лития поток нейтронов внутри кристалла, дает незначительный вклад в величину сигнала детектора, так как захватное излучение из индия находится в слабо поглощаемой области энергий от 4.5 до 8 МэВ. Основной вклад в величину сигнала детектора дает поглощение на изотопе лития, в результате которого возникают две короткопробежные электрически заряженные частицы - $\alpha$-частица и тритий, суммарной кинетической энергией $Q=4.8 \mathrm{MэB}$.

Несложные рассуждения и учет незначительной протяженности поглощающей среды приводят к следующему выражению для количества нейтронов, захватываемых ядрами изотопа лития в единице объема в единицу времени:

$\Delta F_{1} / d=F_{0}\left\{1-\exp \left[-\left(\sigma_{1} n_{1}+\sigma_{2} n_{2}\right) d\right]\right\} \sigma_{1} n_{1} /\left[d\left(\sigma_{1} n_{1}+\sigma_{2} n_{2}\right)\right]$.

Тогда скорость генерации электронно-дырочных пар будет равна

$$
\Delta G=\frac{F_{0}\left\{1-\exp \left[-\left(\sigma_{1} n_{1}+\sigma_{2} n_{2}\right)\right]\right\} Q \sigma_{1} n_{1}}{\varepsilon d\left(\sigma_{1} n_{1}+\sigma_{2} n_{2}\right)},
$$

где $\varepsilon-$ энергия образования одной электронно-дырочной пары в кристалле $p$-T1InSe 2 .

Стационарная же концентрация $p$ генерированных в образце дырок будет равна произведению скорости генерации $\Delta G$ на время жизни неравновесных носителей $\tau$ [9]:

$$
p=\Delta G \tau=\frac{\tau F_{0}\left\{1-\exp \left[-\left(\sigma_{1} n_{1}+\sigma_{2} n_{2}\right) d\right]\right\} Q \sigma_{1} n_{1}}{\varepsilon d\left(\sigma_{1} n_{1}+\sigma_{2} n_{2}\right)} .
$$

Значение величины потока $F_{0}$ в эксперименте было определено известным методом активации фольг с привлечением нейтронно-активационного анализа.

Численное значение стационарной концентрации неравновесных носителей заряда можно определить по величине сигнала $i$ детектора из выражения

$$
i=e p \mu s U / d,
$$

где $e, \mu$ и $s-$ соответственно заряд электрона, подвижность неравновесных носителей тока в кристалле и площадь электродов полупроводникового чувствительного элемента, $U$ - рабочее напряжение детектора.

Значения энергии образования электронно-дырочной пары в $p$-T1InSe 2 , времени жизни и подвижности неравновесных носителей заряда взяты из [10]. Среднее значение величины сечения захвата $\sigma_{1}$ по спектру нейтронов в импульсе принято равным 700 барн, а для $\sigma_{2}-200$ барн. Количество атомов индия в единице объема кристалла составляет $0.9 \cdot 10^{22} \mathrm{~cm}^{-3}$.

Численные значения $p$, рассчитанные с помощью выражения (6) для двух конкретных детекторов с известными размерами чувствительного элемента и измеренной амплитудой отклика, были сопоставлены с выражением для $p$, полученным из (5).

Это сопоставление расчета с экспериментом проводилось следующим образом. В (5) с учетом численного значения $p$ из (6) для конкретных образцов подставляли значения $n_{1}$, близкие справа и слева к значению $n_{1}=(1-1.2) \cdot 10^{21} \mathrm{~cm}^{-3}$, которое было определено по количеству электрического заряда, пропущенного через ячейку в процессе ЭИК. Вычисленные из (5) значения потока $F_{0}$, сравнивались с фактическим значением потока: $F_{0}=2.1 \cdot 10^{8} \pm 15 \%$ нейтрон $/$ см $^{2} \cdot$ с. Совпадение имело место при величине $n_{1}=2.1 \cdot 10^{21} \mathrm{~cm}^{-3}$, что меньше чем в 2 раза отличается от приведенного выше значения.

Совпадение этих величин может в данном случае считаться хорошим и показывает, что метод ЭИК, примененный в настоящей работе, эффективен для кристаллов $p$-TIInSe . $_{2}$

Добавим, что осциллограмма сигнала детектора позволяет, при известной времяпролетной базе, определить энергию нейтрона в любой точке временно́й оси осциллограммы. В частности, из приведенных осциллограмм можно определить, что в распределении тепловых нейтронов данного источника максимум приходится на энергию $\sim 0.065$ эВ.

\section{Заключение}

Как уже упоминалось, серьезным препятствием для увеличения количества вводимого в кристалл $\mathrm{TlInSe}_{2}$ лития являются механические микродефекты, воз- 
никающие в образце при разделке кристаллического слитка. Использованный нами детекторный материал с относительно небольшим сопротивлением, $(0.5-1.0) \cdot 10^{6}$ Ом · см, обладающий повышенной токовой чувствительностью, уступает в механических качествах более высокоомному материалу $\left(\sim 1 \cdot 10^{7} \mathrm{OM} \cdot \mathrm{cm}\right)$, менее подверженному механическим повреждениям. Поэтому, помимо совершенствования средств разделки кристаллического слитка, предполагаем использовать для изготовления детекторов и высокоомный T1InSe 2 .

Авторский коллектив выражает признательность С.С. Павлову за полезные советы и измерение величины нейтронного потока на экспериментальном канале реактора, а также Ю.Б. Гурову за ценные рекомендации при редактировании статьи.

\section{Список литературы}

[1] Ю.И. Алексеев. ПТЭ, № 2, 177 (2008).

[2] Ю.И. Алексеев. ПТЭ, № 6, 37 (2005).

[3] К.Д. Товстюк. Полупроводниковое материаловедение (Киев, Наук. думка, 1984) с. 211.

[4] J. Rouxel. Alkali Metal Intercalation Compounds of Transition Metal Chalcogenides; TX2, TX3 and TX4 Chalcogenides. In: Intercalated Layered Materials, F.A. Levy, ed. (D. Reidel, Dordrecht, Netherlands, 1979) p. 201.

[5] И.В. Алексеев. Неорг. матер., 26 (7), 1401 (1990).

[6] И.В. Алексеев. ФТП, 32 (5), 588 (1998).

[7] Патент РФ № 2135615, дата публикации 27.08.1999.

[8] З.Д. Ковалюк, М.Н. Пырля, А.И. Середюк, К.Д. Товстюк. Неорг. матер., 21 (10), 1652 (1982).

[9] С.М. Рывкин. Фотоэлектрические явления в полупроводниках (М., Гос. изд-во физ.-мат. лит., 1963) с. 49.

[10] И.В. Алексеев. ФТП, 28 (7), 1401 (1990).

Редактор Г.А. Оганесян

\section{The neutron detector based on crystals $\mathrm{TIInSe}_{2}$, intercalated lithium isotope}

\section{I.V. Alekseev, E.A. Goremychkin, N.A. Gundorin,} A.V. Petrenko, I.L. Sashin

Joint Institute for Nuclear Research, 141980 Dubna, Russia

Abstract Explore the possibility of increasing the sensitivity of the neutron detector based on a semiconductor $\mathrm{TlInSe}_{2}$ by introducing a crystal of lithium isopope ${ }^{6} \mathrm{Li}$. The introduction was carried out by electrochemical intercalation of aqueous and non-aqueous solutions of $\mathrm{LiCl}$, and the melt eutectic $\mathrm{LiCl}-\mathrm{KCl}$. The efficiency of intercalation of electrochemical method of $\mathrm{LiCl}$ solution in propylene carbonate, conducted along the axis „a“ of the crystal (along the chains of stronf coupling). Reached concentration of lithium was administered $(1-1.2) \cdot 10^{21} \mathrm{~cm}^{-3}$, which is approximately four times the increased sensitivity of the detector, which amounted to $\sim 1.2 \cdot 10^{-14} \mathrm{~A} \cdot \mathrm{cm}^{2} \cdot \mathrm{s} /$ neutron. Good agreement between the calculated and the actual value of the sensitivity of the detector. 